\title{
TSGN (Edelman) et apprentissage de la lecture
}

\section{Edelman (TNGS) and learning process of reading}

\author{
Jean-Claude Sallaberry ${ }^{1}$ \\ ${ }^{1}$ Professeur émérite à l'Université de Bordeaux — IMS-CNRS, UMR 5218/ISCC, équipe CIH (cognitique et ingénierie \\ humaine), jean-claude.sallaberry@ims-bordeaux.fr
}

RÉSUMÉ. Après un rappel des six paradigmes de l'apprentissage, un des aspects de l'apport d'Edelman est explicité. Son application à l'apprentissage de la lecture montre l'importance de le lier à l'écriture.

ABSTRACT. After mentioning the six paradigms of the learning process, one of Edelman contribution to the theory is explained. Its application to the learning process of reading shows how important it is to link it with writing.

MOTS-CLÉS. Geste, représentation, cartographie neuronale, apprentissage de la lecture.

KEYWORDS. Gesture, representation, neuronal cartography, learning to read.

\section{Introduction}

La modélisation sur laquelle nous allons nous appuyer pour parler d'apprentissage de la lecture est due à Edelman. Mais pour en percevoir la pertinence et l'originalité, il est nécessaire de rappeler le contexte que constituent les six grands paradigmes de l'apprentissage apparus au XXe siècle. Ce sera l'objet du $\S$ I. Ce n'est qu'ensuite, au $\S$ II, que nous présenterons l'apport d'Edelman. Le $\S$ III pourra alors en tirer les conséquences quant à l'apprentissage de la lecture.

\section{Les modélisations scientifiques de l'apprentissage}

Les réflexions sur la question “qu'est-ce qu'apprendre ?" ou "qu'est-ce que connaître ?" ont débuté avec la philosophie. Mais ce n’est qu'au cours du XXe siècle que des tentatives de modélisation

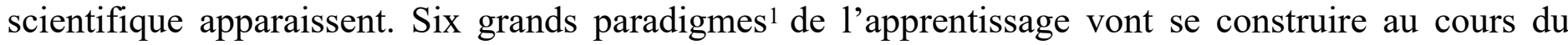
siècle.

\subsection{Le paradigme " associassionniste »}

$\mathrm{Au}$ début du siècle, la psychologie outre-atlantique est dominée par un courant nommé behaviorisme ${ }^{2}$. Sa logique consiste à étudier la liaison entre les éléments d'une situation et les réponses d'un sujet - d'où le schéma S-R ${ }^{3}$. En ce qui concerne l'apprentissage, ce courant s'appuie sur un

\footnotetext{
${ }^{1}$ Un paradigme une théorie suffisamment élaborée, formalisée en tant que "logique“ de modélisation. L'expression "logique de modélisation" exprime à la fois le sens de paradeigma ( $\pi \alpha \rho \alpha \delta \varepsilon \imath \gamma \mu \alpha$, modèle) et la conception du paradigme que propose Kuhn (1962), pour caractériser un mouvement scientifique « établi » (institutionnalisé). Dans le domaine de la mécanique, on peut ainsi parler du paradigme de la mécanique classique (que le formalisme adopté soit newtonien ou lagrangien), du paradigme de la mécanique relativiste, du paradigme de la mécanique quantique.

${ }^{2}$ La science du XIXe siècle, se référant au positivisme, ne peut étudier que des objets positifs, c'est-à-dire réels. Ce caractère de réalité, pour la psychologie, présente une difficulté : si le siège de la pensée et du psychisme semble bien être le cerveau, le fonctionnement de ce dernier reste opaque (d'où l'expression de « boite noire »). La psychologie pense alors pouvoir se constituer en tant que science en étudiant le comportement (behavior) — conçu comme objet réel, observable, du psychisme.

3 « La première orientation est celle des théories de l'apprentissage que l'on qualifie souvent de théories stimulus-réponse ou situationréponse ou S-R. Un lien associatif de plus en plus étroit (liaison conditionnelle, habitude) se forme entre un stimulus et une réponse dont la conjonction s'accompagne plusieurs fois d'un événement agréable, essentiellement la réduction d'un besoin (renforcement). Avec beaucoup de nuances, cette orientation est celle de Thordike, celle de Pavlov, et celles de behavioristes comme Watson... » (Reuchlin, 1977, p.147) 
empirisme associationniste ${ }^{4}$. Dans cette logique de modélisation, un sujet construit l'idée de cercle à partir de l'observation des divers objets ronds qu'il rencontre, et par association de ces différents objets.

Pour Thorndike par exemple, l'apprentissage consiste à établir une relation entre une situation - ou un stimulus - S (le chat est dans la cage et a faim) et la réponse (appuyer sur le loquet de la grille, ce qui permet d'atteindre la nourriture). Les expériences ainsi menées mettent le plus souvent en scène des animaux, dans une situation où ils doivent soit obtenir une gratification (nourriture), soit éviter une souffrance (décharges électriques), en agissant sur un élément du dispositif (levier, pédale...). On constate régulièrement une diminution de la durée écoulée entre le stimulus (la mise en situation) et la réponse lorsqu'on répète l'expérience - d'où la possibilité de tracer une courbe d'apprentissage. La conclusion habituelle est qu'il y a apprentissage, assorti d'un «renforcement» dû aux répétitions. L'application directe de cette théorie au sujet humain a produit l'enseignement programmé, qui préside encore à la conception de la plupart des logiciels d'apprentissage.

Dans une telle conception, un sujet construit l'idée de causalité en associant deux phénomènes qu'il observe habituellement en tant que successifs - le caractère de nécessité viendrait de l'expérience, autrement dit de l'environnement ${ }^{5}$.

\subsection{La théorie de la forme - le paradigme "gestaltiste »}

Une école (germanique) fonde un autre paradigme, nommé gestalt-theorie (théorie de la forme). Ses expériences sont également animalières, mais privilégient des primates plutôt que des rats. Un grand singe se trouve par exemple dans une cage, au «plafond» de laquelle est accroché un régime de bananes. L'animal bondit énergiquement pour décrocher la nourriture. La hauteur ayant été réglée pour que les fruits soient hors d'atteinte, il n'y parvient pas. Et puis soudain, après avoir repris son souffle, le singe utilise une caisse et un bâton placés dans la cage depuis le début. Il décroche alors l'objet convoité. Le caractère soudain de la solution amène les tenants de ce paradigme à rejeter toute hypothèse d'association, qui s'inscrit dans la durée.

Pour reprendre l'exemple du cercle, le paradigme gestaltiste prend le contre-pied du précédent. C'est parce que le sujet possède la « forme » cercle qu'il reconnaît les objets ronds. En ce qui concerne la causalité, il faut que "l'idée de cause" existe a priori chez un sujet pour qu'il établisse un lien de causalité entre deux événements ${ }^{6}$. Ainsi, pour les psychologues qui se rattachent à l'école de la Forme, « le problème est une structure, la solution une autre structure. Résoudre le problème consiste à passer d'une structure à l'autre par une restructuration, une réorganisation qui concerne essentiellement le champ perceptif. » (Reuchlin, 1977, p.214). Les données de l'environnement ne s'imposent pas au sujet sous forme d'éléments, mais lui apparaissent d'emblée structurées, organisées dans des formes - qui ne sont pas réductibles à la juxtaposition des éléments. Lorsqu'il y a restructuration (du champ de perception) cette réorganisation est brusque (insigth).

\subsection{La théorie (et le paradigme) opératoire}

Piaget pense que la théorie associationniste est une genèse (il y a construction de la connaissance) sans structure (du sujet), et que la théorie de la forme s'appuie sur des structures (les formes), mais sans genèse (les formes sont là). Il va tenter de synthétiser (ou de recadrer) les deux théories, en avançant que le sujet interagit avec l'environnement à l'aide de structures, qu'il complexifie et perfectionne au fur et à mesure de ces interactions. Il développe le concept de structure au point d'en

\footnotetext{
4 « Ce rôle considérable et essentiel joué dans l'évolution de la vie psychologique par l'association, sa liaison étroite avec l'habitude, facteur capital de l'adaptation biologique, nous aident à comprendre comment certains psychologues appelés pour cela associationnistes ont eu l'idée d'expliquer par l'association toute la vie psychologique ; » (Rey, 1919, p.122)

5 De ce point de vue, les théories associassionistes assument une filiation vis-à-vis de l'empirisme, mais aussi de la théorie de Lamarck.

6 Les théories aprioristes sont alors à référer non seulement à Kant, mais aussi à un néodarwinisme (l'adaptation est « endogène »). 
proposer la généralisation $(1968)^{7}$. S'appuyant sur la notion de schème ${ }^{8}$, la théorie opératoire qu'il bâtit est sans doute ainsi nommée pour l'importance qu'elle accorde, à juste titre, aux opérations que le sujet fait subir aux objets. Chaque fois qu'un sujet fait rentrer un objet dans l'un de ses schèmes d'action processus d'assimilation de l'objet - on peut considérer que l'objet subit une opération'. Mais il est possible de justifier ce qualificatif par la conception opératoire, ou opérationnelle, que ce paradigme propose de l'intelligence. En effet, penser cette dernière comme capacité d'action sur les objets, c'est faire accéder la notion au stade de concept ${ }^{10}$, par opposition à l'imprécision d'autres définitions ${ }^{11}$, par opposition au caractère immanent des soi-disant critères auxquels renverraient les significations courantes du terme. La conséquence immédiate de la définition opératoire est la possibilité de penser une intelligence en actes, capacité d'action sur des "objets" concrets. Établir une correspondance terme à terme pour vérifier l'égalité de quantités de deux séries d'objets - vérifier en mettant "face à face" des boules rouges et des boules blanches "qu'il y en a autant" - correspond bien à l'intelligence. De même la capacité à varier des déplacements pour atteindre le même point dans l'espace.

Au cours de son développement, il y a un moment où le sujet n'a plus besoin de "faire", d'agir concrètement pour penser - le moment par exemple où il peut penser les déplacements, sans les réaliser, pour en tirer des conclusions. Le sujet, alors, peut "agir en pensée“. Il se représente les objets et ses actions sur ces objets et peut agir sur ses représentations. Et toute la puissance conceptuelle de la définition opératoire de l'intelligence tient dans ce renvoi symétrique qui est aussi une oscillation de notre fonctionnement cérébral, de notre pensée : "penser en agissant" et "agir en pensée". Ainsi, la complexification des structures du sujet l'amène à passer d'une intelligence "concrète" (sensorimotrice) à une intelligence abstraite (fondée sur les représentations), puis à une intelligence formelle (fondée sur les représentations et les opérations). Le sujet construit son savoir tout en perfectionnant ses structures - au niveau théorique comme au niveau épistémologique, il s'agit d'un

\section{constructivisme.}

\subsection{Les théoriciens de la médiation (paradigme constructiviste-interactionniste)}

Le reproche le plus courant adressé à Piaget est celui d'une théorie qui décrit un sujet au développement "isolé" - ce dont Piaget se défend. Il est vrai que si les structures du sujet se complexifient à partir de leur interaction avec l'environnement, on ne voit guère intervenir d'autres personnages, en "chair et en os". Les positions de Wallon, Vygotsky, Bruner vis-à-vis de la théorie de Piaget convergent sur l'importance de l'intervention des autres, et notamment des adultes, dans le développement de l'enfant ${ }^{12}$. Certains auteurs, comme Doise et Mugny, en s'appuyant sur la théorie opératoire tout en tenant le plus grand compte de l'interaction sociale, vont privilégier le «conflit socio-cognitif $»^{13}$.

\footnotetext{
7 Il propose de caractériser la structure par trois critères : totalité, transformations, autoréglage - pour plus de précisions, cf. Sallaberry \& Claverie, 2018.

8 Rappelons que le plus simple pour définir un schème est de le considérer comme une série d'actions permettant d'atteindre un objectif. Par exemple, le schème de nidation chez les oiseaux comprend la série d'actions qui sont nécessaires à l'élaboration d'un nid. Vergnaud (1995, p.66) en donne une définition plus structurale, en proposant de le considérer comme "l'organisation invariante de la conduite pour une classe de situations donnée."

9 Schématiquement, une opération est une "action plus la réversibilité" — une action pensée comme étant réversible.

10 Un concept rassemble tous les sens du terme, en les dépassant. Il permet de concevoir ce qui jusque là était seulement évoqué, approché (Lefebvre, 1980). Ici, l'une de ses caractéristiques essentielles est d'avoir des bords nets : possibilité de repérer clairement ce qui est ou n'est pas décrit par le concept, possibilité de connaitre les situations concernées - cf.Sallaberry,1996.

11 Concevoir l'intelligence comme capacité d'adaptation, comme on l'entend souvent, est intéressant mais moins opérationnel. Les situations pouvant varier, sinon à l'infini, du moins beaucoup, à quel type de situation fait-on référence quand on parle d'adaptation ? Par ailleurs, on peut aussi se demander de quel type d'adaptation il s'agit ou, plus précisément, quels sont les critères qui permettent de classer les adaptations possibles. Ne dépendent-ils pas, en fait, des situations ?

12 On peut ainsi, de ce point de vue, considérer ces trois auteurs comme des "théoriciens de la médiation".

13 « Nous ferons l'hypothèse... que c'est au travers de conflits de centration que s'effectue cette élaboration cognitive. L'idée d'un conflit socio-cognitif comme processus par lequel s'actualiserait le développement cognitif relève d'une conception à la fois interactionniste et constructiviste : c'est en effet à partir d'expériences cumulatives et progressives que les sujets élaborent des ๑) 2018 ISTE OpenScience - Published by ISTE Ltd. London, UK - openscience.fr Page $\mid 3$
} 
La position de Vygotsky, même si elle peut être considérée comme proche puisqu'il s'agit toujours d'un « interactionnisme social » ${ }^{14}$, est plus radicale, puisque pour lui «la vraie direction du développement ne va pas de l'individuel au social, mais du social à l'individuel» (1934) ${ }^{15}$. Son hypothèse est ainsi que la pensée « collective » précède la pensée « individuelle », puisque vis-à-vis de la situation d'apprentissage un concept existe déjà au niveau collectif lorsqu'il est proposé à la construction par un sujet. Il construit la notion de $"$ zone proximale de développement $»^{16}$, pour repérer la différence entre le développement « déjà réalisé » et celui qui devient possible dès lors que l'adulte aide l'enfant ${ }^{17}$. Si l'activité est centrale, elle est non seulement « individuelle-cognitive » mais aussi « sociale-interactive ». Il considère aussi que cognition et émotion sont indissociables (1998).

Pour Wallon et Bruner, l'acte est également central, mais il est conçu en boucle, l'effet produit dans le contexte qui est celui de l'acte — ayant un rôle " organisateur » sur l'acte ${ }^{18}$. Plus récemment, Bruner insiste, outre sur l'importance de la médiation d'autrui (1987), sur le caractère structurant de la culture pour la pensée comme pour le développement (1996), en prônant une «psychologie tournée vers la culture » (1997, p.34).

C'est dans ce contexte (si les écrits de Vygotsky ne sont connus que plus tard, Wallon est un collègue de Piaget, Bruner a travaillé avec Piaget et publié avec lui ${ }^{19}$ ), du moins de ce côté-ci de l'Atlantique, que de nouveaux outils de modélisation de la cognition, et donc de la pensée vont apparaître.

\subsection{Paradigme cognitiviste (fort)}

Un paradigme radicalement nouveau prend sa source dans le mouvement cybernétique ${ }^{20}$. Son hypothèse fondatrice peut s'énoncer : «Connaître, c'est effectuer, sur des représentations, des manipulations réglées. » (Dupuy, 1994, p.22) ${ }^{21}$. Reprise par Newell et Simon (1972), par tout un pan

instruments cognitifs nouveaux, au travers d'interactions lors desquelles le sujet est essentiellemnt actif... Le progrès cognitif, qui a été décrit comme un processus de décentration, serait surtout suscité par la nécessité de coordonner des centrations opposées dans une action commune. » (Doise, Deschamps, Mugny, 1978, p.255)

14 «Cette idée de la genèse sociale de la pensée, héritée à la fois de Spinoza et du marxisme, et déjà présente dans un article de 1924, est sans doute l'élément nodal de la pensée de Vygotsky, et celui par lequel il se distingue radicalement d'auteurs comme Piaget :

- le concept central de la psychologie est celui d'activité, définie comme l'unité d'analyse intégrant caractéristiques socialesinteractives et individuelles-cognitives des conduites ;

- la coopération sociale dans l'activité se réalise au moyen d'instruments, au rang desquels les signes verbaux jouent un rôle primordial ; c'est par l'intériorisation progressive de ces instruments de coopération que se construit la pensée consciente, qui prend en charge et "régule" les autres fonctions psychiques. Au terme de ce processus, la conscience devient "un contact social avec soi-même".

Il résulte de ces thèses fondatrices de l'interactinonisme social que les instruments sont des moyens de contact avec le monde extérieur aussi bien qu'avec soi-même (avec sa propre conscience) et que c'est donc au travers de leur étude que se réalisera l'unification de la psychologie. » (Bronckart, 1985, p.14).

15 «Chaque fonction psychique supérieure apparaît deux fois au cours du développement de l'enfant : d'abord comme activité collective, sociale et donc comme fonction interpsychique, puis la deuxième fois comme activité individuelle, comme propriété intérieure de la pensée de l'enfant, comme fonction intrapsychique. » (Vygotsky, 1985, p.111)

16 1985, p. 107.

17 « Ce que l'enfant est capable de réaliser avec l'aide de l'adulte délimite sa zone proximale de développement. » (1985, p.109).

18 «... Bruner... soutient que l'effet, c'est non seulement le résultat empirique et la sanction extrinsèque que celui-ci apporte à l'activité, mais aussi (et surtout) la transformation de l'acte lui-même, transformation au terme de laquelle l'acte « se trouve bientôt supplanté par une nouvelle configuration d'action qui peut fort bien avoir pour composante l'acte qui vient d'être maîtrisé. » » (Deleau, 1983, p.23).

$19 C f$. Bruner 1958 et Piaget 1958. Bruner (1958) décrit la perception comme fondamentalement liée à un acte de catégorisation (si on veut résumer grossièrement, la perception correspondrait à représentation plus catégorisation). Piaget semble avoir une position plus nuancée, tout en s'appuyant sur Bruner. Ce dernier rapproche perception et représentation, puisqu'il va jusqu'à accorder une "fonction représentative" à la perception. Piaget (1958) prend en compte ce point de vue. ( $c f$. Sallaberry 2015c ).

20 Cela ne signifie pas, bien entendu, qu'il n'y ait pas de lien ou de contact entre la cybernétique et le structuralisme. Témoins l'intérêt de Piaget pour les travaux de Simon, ainsi que l'émergence de la théorie des systèmes dont Le Moigne (1990) souligne la double origine : cybernétique et structuralisme.

21 Dupuy précise (p.22) : «L'essentiel, c'est la nature logique des manipulations et des règles en question... L'époque qui précède immédiatement l'histoire que nous allons conter (il s'agit de celle des conférences Macy) a produit des modèles mathématiques tant du système nerveux que des circuits électriques. Il aura cependant fallu le génie de McCulloch et Pitts d'un côté, de Shannon de l'autre, (c) 2018 ISTE OpenScience - Published by ISTE Ltd. London, UK - openscience.fr 
de la recherche en psychologie - tendance bien représentée par Richard (1990) - elle correspond actuellement au "paradigme cognitiviste fort", l'un des deux grands courants des sciences de la cognition. A partir du constat que trois diodes 22 , suivant leur montage, peuvent réaliser les fonctions logiques élémentaires (telles que "et", "ou“...), il semble possible de modéliser le fonctionnement du cerveau et de la pensée. Cela, en poussant plus ou moins loin la comparaison avec le fonctionnement de l'ordinateur, comparaison qui, pour le confort, devient la "métaphore de l'ordinateur" 23 . Cette logique de modélisation ('penser revient à manipuler des informations', conception étendue à 'penser c'est computer des représentations'), qui s'est propagée de manière remarquable en dehors des sphères de la recherche, est devenue banale - les écrits s'adossant à la comparaison cerveau-ordinateur, ou pensée-traitement de l'information ne se comptent plus.

Dans cette perspective, un courant de l'intelligence artificielle s'est développé ; à partir de cette conception, les "représentations" (utilisées dès lors pour être "calculées"), doivent, bien entendu, répondre aux règles de la logique formelle ${ }^{24}$. On ne peut donc travailler avec des propositions du genre "je veux a mais aussi non-a“. Par ailleurs, la tentative de réalisation de systèmes experts montre qu'il est bien difficile de bâtir un logiciel d'efficacité comparable à celle d'un expert en chair et en os.

Nous avons signalé l'apparition de ce paradigme dans le contexte européen. Pour ce qu'il en est du contexte outre-atlantique, et de la "comparaison - assimilation cerveau/ordinateur" que nous avons signalée, nous laissons la parole à Bruner, qui critique vivement : «On a très vite identifié les processus cognitifs aux programmes que l'on pouvait faire "tourner" sur un ordinateur... Ce nouveau réductionnisme a autorisé un libéralisme étonnant. Les partisans de l'ancienne théorie de l'apprentissage par stimuli-réponses, ceux de la mémoire par associations, tous ont pu trouver place dans la révolution cognitive; il leur a suffi d'appliquer à leurs vieux concepts la terminologie en vogue, empruntée au traitement de l'information. Il n'était même pas nécessaire d'adopter les concepts de "signification" ou "d'opérations mentales"; on remplaçait "stimulus" par "entrée" et "réponse" par "sortie"; on gommait tout ce qu'il y a d'affectif en évoquant "le feed-back contrôlé des sorties du système"; et le tour était joué. Tout programme informatique devenait un "esprit".» (1997, p.22). Il faut rappeler que ce qui a été désigné par la "métaphore de l'ordinateur" s'appuie sur une idée féconde de Turing, qui estimait que l'on pouvait comparer deux systèmes qui exécutent une tâche similaire. Ainsi, l'ordinateur est capable d'effectuer des calculs, le cerveau aussi. Nous pensons, à la suite de Turing, qu'il est tout à fait pertinent de comparer, sur cette base, cerveau et ordinateur. Mais comparer n'est pas identifier...

\subsection{Paradigme connexionniste}

Un second courant des sciences de la cognition met l'accent sur les connexions entre neuronesd'où son appellation de connexionnisme. L'apprentissage correspond à des modifications du réseau liées au degré d'activité corrélée entre les neurones. Si deux neurones s'activent au même moment, leur lien est renforcé ; dans le cas contraire, il est diminué — cet énoncé correspondant au principe de Hebb.

pour comprendre que la modélisation pertinente était en fait de type logique — donc que l'on pouvait décrire en termes logiques le fonctionnement de certains systèmes matériels, mais qu'inversement ces systèmes matériels pouvaient se représenter comme réalisant, voire incarnant la logique, cette forme supérieure de la pensée. »

22 Ce composant pouvant pour le courant être "passant" ou "non passant", il peut exhiber deux états (qui peuvent être codés par 0 et 1) et peut donc être considéré comme neurone "formel" (ou artificiel).

23 Combien de recherches, avant de s'appuyer sur l'hypothèse de l'existence de deux types de mémoire — mémoire à court terme et mémoire à long terme — se posent sérieusement la question de la probabilité de repérage expérimental des supports neuronaux correspondants, au vu des recherches actuelles en neurosciences animale ou humaine?

24 Repérable à ses trois principes :

identité : a est a

- $\quad$ non-contradiction : si p est une proposition et non-p sa contradictoire, $\mathrm{p}$ et non-p ne peuvent être ni toutes les deux vraies ni toutes les deux fausses dans le même système logique

tiers exclus : si on peut comparer la proposition $q$ à $p$, ou bien $q=p$, ou bien $q=$ non- $p$

(c) 2018 ISTE OpenScience - Published by ISTE Ltd. London, UK - openscience.fr 
Dans cette logique de modélisation, on peut observer, à partir d'un certain niveau de complexité d'un réseau), des propriétés émergentes ${ }^{25}$. Avec une telle interprétation, la cognition est l'émergence d'états globaux d'un réseau de composants simples (neurones naturels ou formels). La conscience, le langage, la pensée, sont alors des propriétés émergentes du fonctionnement du système nerveux. Conséquence immédiate, ces propriétés sont relativement "distribuées" (et non "localisées") ${ }^{26}$. Elles sont à concevoir comme résultant de l'interaction entre "agents" (neurones ou réseaux de neurones) ${ }^{27}$.

\subsection{Commentaires et développements plus récents}

Chacun des paradigmes propose bien sûr des outils de modélisation, que l'on a intérêt à concevoir comme complémentaires, même lorsqu'ils semblent s'opposer. Par exemple, l'associationnisme insiste sur l'intérêt de l'entraînement et le principe de Hebb permet d'interpréter l'efficacité de l'entraînement.

Ce rappel sur les six paradigmes est important pour situer le contexte de notre propos, mais aussi pour faire comprendre qu'il serait indispensable de faire réfléchir les futurs enseignants sur ces diverses modélisations de l'apprentissage, considérées comme possédant des pertinences complémentaires.

\subsubsection{Varela et Edelman}

Ces deux grands chercheurs, à partir du paradigme connexionniste ${ }^{28}$, amènent des outils de modélisation remarquables ${ }^{29}$.

Varela invente l'idée du processus d'autopoièse pour des systèmes qui se produisent eux-mêmes ${ }^{30}$ - qu'il va donc qualifier d'autopoiétiques. C'est un enrichissement considérable, à la fois pour la nomenclature des systèmes (puisqu'il s'agit d'une nouvelle classe de systèmes) et pour la notion d'émergence, puisque ce n'est dès lors pas seulement une nouvelle propriété qui émerge au sein d'un système, mais le système lui-même qui se reconfigure et se reconstitue en parmanence. Un autre article se chargera de présenter plus amplement les travaux de Varela.

Le $\S$ II présente quelques aspects des propositions d'Edelman.

\subsubsection{Changeux}

Changeux (2002) propose ${ }^{31}$, pour l'apprentissage et la cognition, une conception qui repose sur l'élaboration puis la sélection, par le sujet humain, de pré-représentations, au cours de jeux cognitifs entre le sujet et le monde extérieur. Il se situe clairement du côté du connexionnisme (cf. note 26). Un aspect particulièrement intéressant de l'ouvrage est le lien établi entre le développement du cerveau et les interactions sociétales. Si Bruner intitule l'un de ses ouvrages "Car la culture donne forme à l'esprit", ce passage de Changeux pourrait se résumer par la formule 'Et la culture donne forme au cerveau'. Cela sera développé dans un prochain article.

25 Pour plus de détails, on se reportera à Dupuy (1994), Andler (1992), Edelman (1992), Varela (1989).

26 «En accord avec la manière dont nous avons envisagé la genèse et la stabilisation des assemblées de neurones, il paraît au contraire plus raisonnable d'envisager que la "trace" des objets de mémoire se trouve distribuée sur l'ensemble du cortex et, pourquoi pas, sur une grande partie de l'encéphale. » (Changeux, 1983, p. 224)

27 On peut concevoir un agent comme assumant un rôle fonctionnel (il assure une fonction). On peut généraliser en le considérant comme un sous-système, par définition moins complexe que le système global. Cette idée d'interaction ou de coopération (entre soussystèmes), déjà présente chez Gazzaniga (même s'il reste très influencé par la première cybernétique puisqu'il ne peut concevoir qu'il n'y ait pas de sous-système de pilotage) est notamment développée par Minsky (1988).

28 Varela enrichit les notions de distribution et d'émergence, Edelman mène une critique serrée du paradigme cognitiviste fort.

${ }^{29}$ Varela (dans l'ouvrage qu'il signe avec Thomson et Rosch, 1993) référencie Edelman. Ce dernier ne cite pas Varela, mais cite et référencie Rosch.

30 Пoıฑбı (poièsis) signifie, en grec ancien, 'action de faire', d'où 'I création ; II fabrication, confection ; III action de composer des œuvres poétiques' (Bailly).

31 Présente dès 1983, elle se précise et se développe dans l'ouvrage de 2002.

(c) 2018 ISTE OpenScience - Published by ISTE Ltd. London, UK - openscience.fr 


\section{Edelman et la TSGN}

\subsection{Groupes neuronaux et cartes neuronales}

Edelman (1992) élabore une théorie qu'il nomme la TSGN (théorie de la sélection des groupes neuronaux) ${ }^{32}$. Dans la complexité de notre système nerveux, il propose de discerner les groupes neuronaux et les cartes neuronales.

Les groupes neuronaux sont fondés sur une sorte de propagation de l'excitation due à la proximité. Les neurones voisins d'un neurone excité (ou activé) ont des chances de l'être aussi, mais construisent une dynamique propre :

« Nous pouvons donc résumer la situation en disant que, en général, aucun neurone n'est sélectionné isolément dans une carte, aucun neurone n'est connecté de façon réentrante à un unique neurone d'une autre carte; qu'aucun neurone isolé ne présente les propriétés qu'il présente au sein d'un groupe. » (p.136)

Les cartes neuronales correspondent à une fonctionnalité (nécessaire à l'accomplissement d'un geste, par exemple). Les cartes interagissent grâce à un processus appelé réentrée, qui amène l'émergence de propriétés nouvelles :

«... la propriété de réentrée autorise ce que j'appelle une synthèse récursive : non seulement les événements sont corrélés topographiquement sur un ensemble de cartes sans l'aide d'un quelconque superviseur, mais des propriétés sélectives nouvelles émergent au cours du temps par réentrées successives et récursives à travers les cartes. » (p.138)

Lorsqu'un sujet agit, il met ainsi en jeu à la fois des groupes neuronaux et des cartes neuronales, cet ensemble étant nommé cartographie globale. Une cartographie globale est une structure dynamique qui couple les sorties d'une multitude de cartes interconnectées de façon réentrante au comportement sensori-moteur.

Remarquons qu'avec la propriété de réentrée nous retrouvons ce qui caracétrise un fonctionnemnt en boucle. Mais en outre Edelman souligne des changements de niveaux, ce qui fait de ces boucles des boucles étranges ${ }^{33}$.

\subsection{Propositions d'Edelman et représentation (d'un geste)}

Edelman ne parle pas directement de représentation ${ }^{34}$, mais de mémoire et de remémoration. Pour résumer ce qui nous semble essentiel, nous allons citer quelques passages, en renvoyant le lecteur à l'ouvrage d'Edelman pour plus de précisions.

\section{A propos de la mémoire}

« Pour clarifier les idées à ce propos, nous allons convenir que, quelle que soit la forme qu'elle adopte, la mémoire sera toujours définie comme la capacité à répéter une performance. » (p.157)

\footnotetext{
32 «Si nous admettons que les fonctions cérébrales sont bâties selon un processus sélectif, nous devons alors être capables de réconcilier la variabilité structurelle et fonctionnelle du cerveau avec la nécessité d'expliquer comment il effectue des catégorisations. » (p.127)

33 Les "boucles autopoiétiques" (voir Varela) sont des boucles étranges - cf. Sallaberry \& Claverie 2018. Cette convergence avec Varela est d'autant plus nette que l'interconnexion réentrante au comportement sensori-moteur ressemble fort à la boucle 'action/perception'.

34 Sauf dans la postface critique, au moment où il aborde la question de l'intentionnalité. « En fait, comme l'a fait remarquer Searle, le contenu sémantique n'a aucun sens en l'absence d'intentionnalité, c'est-à-dire de la capacité de faire référence à d'autres états ou objets. Et pour qu'on en soit capable, il faut que les représentations formelles deviennent des représentations intentionnelles. » (p.367) 
« La TSGN postule que la mémoire consiste plutôt en un renforcement spécifique d'une capacité de catégorisation préalablement établie. Ce type de mémoire est une propriété collective issue des modifications dynamiques survenant continuellement dans les populations synaptiques des cartographies globales - et qui sont du même type que celles qui ont permis à des catégorisations d'avoir lieu au départ. La modification des force synaptiques des groupes dans une cartographie globale constitue la base biochimique de la mémoire. » (p. 157-158)

«Cette mémoire constitue une propriété de l'ensemble du système, bien que son mécanisme de base soit la modification des efficacités synaptiques...»(p.159)

\section{A propos de la remémoration}

«Dans un tel système, la remémoration n'est pas stéréotypée. Sous l'influence de contextes qui se modifient constamment, elle change à mesure que la structure et la dynamique des populations neuronales impliquées dans les catégorisations originelles changent elles aussi. La remémoration fait appel à l'activation de certaines portions - mais pas nécessairement à la totalité - des cartographies globales établies auparavant. Elle peut ainsi donner lieu à des catégorisations semblables à des catégorisations précédentes, mais, à des instants différents, les éléments contribuant à cette réponse seront eux-mêmes différents, et en général ils seront susceptibles d'être modifiés par les comportements survenus entre temps. » (p.158)

\section{La mémoire à nouveau}

«Puisque les catégories perceptives ne sont pas immuables et qu'elles se modifient sous l'effet des comportements de l'animal, la mémoire, vue sous cet angle, résulte d'un processus de continuelle recatégorisation. Par nature, elle fait intervenir des procédures, une activité motrice continuelle et des essais répétés dans divers contextes. Par nature, elle fait intervenir des procédures, une activité motrice continuelle et des essais répétés dans divers contextes. »(p.158)

On pense ici à la boucle 'action-perception' proposée par Varela ${ }^{35}$.

\section{Remémoration et représentation}

La mémoire consistant en la capacité à reproduire une performance, la remémoration d'un geste revient à soit à engager à nouveau le geste réellement, soit à l'engager potentiellement, c'est-à-dire à se le représenter. Et ce que suggère la proposition d'Edelman, c'est que dans les deux cas c'est la même cartographie neuronale globale (ou à peu près) qui va être activée. La première interprétation de cet 'à peu près' consiste à penser que cette deuxième cartographie (celle qui correspond à la représentation $\mathrm{du}$ geste) n'active pas les neurones effecteurs (ceux qui commandent les muscles). Or, dans une présentation de cette modélisation auprès de responsables sportifs et d'entraîneurs (il s'agissait d'une conférence lors d'un colloque), ces derniers ont été fortement intéressés et m'ont appris que les entraineurs exigeaient de leurs sportifs qu'ils pensent à leurs gestes précis d'entraînement durant les déplacements (en car ou en avion, peu importe) qui précèdent les compétitions. Il est clair en effet qu'une telle modélisation justifie cette consigne. Mais en retour l'existence de cette consigne formulée par les entraîneurs constitue un appui de taille quant à la pertinence de cette modélisation.

Détail de convergence : tout en conduisant ma voiture au retour de cette conférence, j'ai décidé de penser aux exercices abdominaux que je pratique lors des matinées "tranquilles". J'ai eu la surprise de constater que cette seule pensée (cette représentation) déclenchait la contraction des muscles concernés 
par cet exercice - contraction plus faible que lors de l'exercice réel, mais contraction tout de même. La cartographie "représentation" active bien y compris les neurones effecteurs.

Illustrons cela sur un autre exemple.

\section{Application à la représentation d'un geste}

Assis devant une table, je saisis un objet, un crayon, par exemple. Je le lève, puis le repose sur la table, à un autre emplacement. Je viens d'agir, l'action consistant en un déplacement d'objet C'est de l'action, c'est du concret (plus précisément, l'objet est réel, concret, l'acte également) ${ }^{36}$. Maintenant, ayant remis le crayon à son emplacement initial, je pense que je déplace le crayon (le même déplacement que précédemment). Ce faisant, je me représente l'action précédente - le déplacement du crayon. Je viens de passer du concret (le geste) à l'abstrait, même si cette abstraction reste très proche du réel. Selon la modélisation d'Edelman, j'ai mis en jeu, pour déplacer le crayon, une cartographie neuronale globale. Ensuite, lorsque je me représente cette action, j'active pratiquement la même cartographie. Telle est la proposition d'Edelman, que l'on peut estimer particulièrement économique. Suggérant en effet que le support neuronal de la représentation est à peu près le même que celui du geste - il s'agit bien sûr de la représentation du geste, elle permet d'articuler geste et pensée, de donner au geste le statut d'une figure de la représentation ${ }^{37}$.

Il est dès lors possible de concevoir (ou de convenir) que l'acte réel constitue une action concrète et que la représentation (de cet acte) est une action abstraite - une action en pensée, qui accompagne le plus souvent l'action concrète (l'homme se "voit" agir, tout en agissant) ${ }^{38}$. Cette perspective fait appel à la conscience, qu'Edelman aborde plus loin dans son ouvrage ${ }^{39}$, de même que la question des capacités conceptuelles ${ }^{40}$. Cet article ne retient pour son propos qu'un aspect du très riche travail de ce prix Nobel.

Si à partir des travaux d'Edelman la représentation "ressemble“ beaucoup à l'action — du point de vue des groupes neuronaux et des cartes neuronales mis en jeu, on peut noter les convergences avec les conceptions de Varela, mais aussi avec la position de Bruner (1958), à propos de l'importance de la catégorisation dans la perception et la représentation ${ }^{41}$. Plus précisément, c'est le substrat neuronal (la cartographie globale - l'état global qui émerge pour Varela) de l'une et de l'autre qui est, sinon strictement le même, du moins semblable.

Comme se souvenir, dans ce type de modélisation, consiste à activer à nouveau une cartographie que l'on a déjà activée (il y a plus ou moins longtemps, et avec une fréquence plus ou moins

\footnotetext{
36 L'inconvénient de l'écrit, c'est qu'il faut faire appel, pour faire comprendre cet acte concret, à la capacité de représentation du lecteur!

37 Cf. Sallaberry \& Claverie, 2018, Chap VI.

38 Bien entendu, il y a des niveaux d'abstraction plus élevés que la simple "image“ du geste — il suffit par exemple de penser à l'équation de la trajectoire du stylo.

39 «Pour voir comment tout cela fonctionne, il nous faudrait plonger dans un océan de détails techniques. Néanmoins, il est important de comprendre quels sont les moyens mis en œuvre par le cortex cérébral et ses appendices pour satisfaire les exigences temporelles et spatiales de la mémoire; Je rappelle que le cortex est une structure constituée de six couches interconnectées, contenant quelque dix milliards de neurones et environ un million de milliards de connexions. Outre le fait qu'il est structuré en cartes aux fonctions distinctes, connectées de façon réentrante et desservant la totalité des modalités sensorielles et des réponses motrices, il est relié à trois structures, que j'appelle les organes de la succession — parce qu'ils interviennent, bien entendu, dans l'organisation des sortes du cerveau. Chacune de ces structures - le cervelet, l'hippocampe et les ganglions de base - traite d'un aspect différent et d'une échelle différente de cette organisation. »(p.161)

40 «La TSGN postule que le développement, au cours de l'évolution, d'aitres cérébrale spécialisées est nécessaire à l'apparition de capacités conceptuelles. » (p.167)

41 Dans ce chapitre qui ouvre l'ouvrage collectif dirigé par Piaget, Bruner développe une conception de la perception qui, si on la résume rudement, correspondrait à la combinaison (ou la coordination) représentation-catégorisation. 
importante) $)^{42}$, on comprend qu'il est beaucoup plus facile de mémoriser si ce qu'on veut se rappeler (une représentation) est associé à un geste.

\section{Application à l'apprentissage de la lecture}

Poursuivre la réflexion entamée - ou si l'on préfère continuer d'utiliser la modélisation précédente — en l'appliquant à la lecture (et à son apprentissage) amène à se demander quelle est la représentation la plus immédiate d'une lettre, d'une syllabe, ou d'un mot et quels sont les gestes qui peuvent y être associés.

Dans l'apprentissage de la langue, celui de la langue orale (le parler) a en général précédé celui de la langue écrite. Dans le cas le plus simple (et le plus courant), un mot a été associé à un objet ${ }^{43}$. La représentation visuelle, l'image de l'objet, a été ainsi reliée à une représentation sonore ${ }^{44}$. Le geste correspondant aux représentations sonores de la langue est celui de la prononciation des mots.

L'apprentissage de la lecture advient ainsi, d'habitude, sur un substrat de connaissance orale de la langue. La représentation d'une lettre, d'une syllabe, d'un mot par une ou plusieurs lettres écrites doit donc s'associer aux représentations sonores. Le geste, nouveau, consiste à écrire (lettre, syllabe, mot...).

Si nous reprenons maintenant l'idée que le geste va faciliter la mémorisation, nous pouvons dire que la représentation (d'un son s'il s'agit d'une lettre ou d'une syllabe, de deux ou plusieurs dans le cas d'un mot) sera d'autant plus facile à mémoriser qu'elle sera associée à un geste, celui qui consiste à écrire (la syllabe ou le mot).

Il est donc essentiel que l'apprentissage de la lecture soit associé à l'écriture.

Or, les enfants écrivent de moins en moins à l'école. Le travail sur fiches s'est généralisé. Gain de temps, diront les enseignants, et d'énergie (ils n'ont plus à écrire au tableau une phrase à travailler, un énoncé de problème...). Ce "gain de temps" apparent ignore que le temps d'écriture d'un énoncé est aussi celui de l'appropriation de l'énoncé. Et lorsqu'il s'agit d'un travail sur la langue, le travail demandé par la fiche comporte en général fort peu d'écriture : il faut écrire un ou deux mots dans une phrase à trous, par exemple. Au passage, cette pratique des fiches fait que nos enfants manipulent un livre bien moins souvent. Le livre devient ainsi un objet moins familier, nouvel inconvénient.

In fine, les élèves sont trop souvent dans des situations dans lesquelles ils écrivent fort peu, ou en tout cas insuffisamment. Cela entraine une perte d'efficacité considérable dans l'apprentissage de la lecture et bien sûr dans celui de la langue (orale ou écrite). Dès lors, par rapport à l'inquiétude légitime que les français peuvent éprouver vis-à-vis de la moindre maîtrise de la langue par les jeunes (évoquée aussi bien par les enquêtes internationales que par les constats des enseignants), point n'est besoin de chercher ailleurs des leviers d'amélioration (ou de remédiation) - c'est ce que j'écrivais (avec moins de détails) en réponse à l'ouvrage (au brûlot) de Carole Barjon ${ }^{45}$. Il est crucial que les enfants écrivent, chaque jour, à l'école — et écrivent suffisamment !

\footnotetext{
42 Cette nouvelle activation est d'autant plus facile que les activations de cette cartographie sont (ou ont été) fréquentes (principe de Hebb).

${ }^{43}$ Cette formulation est bien sûr trop rapide. Mais le propos ici n'est pas de faire le point sur cet aspect ; je renvoie donc à Bruner, 1983 pour plus de précisions.

44 Sibony fait remarquer qu'à l'époque de Mahomet, «il est interdit dans les terres conquises de faire entendre les cloches des églises, représentation sonore du christianisme. » $(2015$, p. 306) C'est une belle évocation des représentations sonores.

45 Sallaberry, 2016.

๑) 2018 ISTE OpenScience - Published by ISTE Ltd. London, UK - openscience.fr 


\section{Conclusion}

Après avoir rappelé les six grands paradigmes de l'apprentissage — élaborés au XXe siècle, nous avons évoqué les aspects du travail d'Edelman susceptibles d'être pertinents vis-à-vis de l'apprentissage de la lecture. Nous avons souligné, à l'aide de cet appui, l'importance cruciale de coordonner, d'articuler cet apprentissage à celui de l'écriture.

\section{Références}

[BOY 96] BOYSSON-BARDIES B.,de, 1996, Comment la parole vient aux enfants, Paris, Odile Jacob.

[BRE 87] BRESSON, F.,1987, Les fonctions de représentation et de communication, in Psychologie, s/d PIAGET, MOUNOUD, BRONCKART, Paris, NRF (Encyclopédie La Pléiade).

[BRO 85] BRONKART, JP., 1985, Vygotsky, une oeuvre en devenir, in Vygotsky aujourd'hui, s/d SNEUWLY \& BRONCKART, Lausanne, Delachaux \& Niestlé.

[BRU 58] BRUNER, JS., 1958, Les processus de préparation à la perception, in Études d'épistémologie génétique s/d J.PIAGET, tome VI Logique et Perception, Paris, PUF.

[BRU 87] BRUNER J.S. , 1987, Comment les enfants apprennent à parler, Paris, Retz.

[BRU 90] BRUNER J.S. , 1990, ... Car la culture donne forme à l'esprit, tr.fr. 1991, Paris, Eshel.

[BRU 96] BRUNER J.S. , 1996, L'éducation, entrée dans la culture, Paris, Retz.

[CHA 83] CHANGEUX JP. (1983), L'homme neuronal, Paris, Fayard.

[CHA 02] CHANGEUX JP. (2002), L'homme de vérité, Paris, Odile Jacob.

[CLA 05] CLAVERIE, B., 2005, Cognitique, Paris, L'Harmattan (Cognition et Formation)

[DAM 99] DAMASIO, A.R., 1999, Le Sentiment même de soi, Paris, Odile Jacob 2002.

[DEL 83] DELEAU, M., 1983, Présentation de, Le développement de l'enfant, savoir faire savoir dire, Paris, P.U.F.

[DOI 78] DOISE W., DESCHAMPS J.C., MUGNY G., 1978, Psychologie sociale expérimentale, Paris Armand Colin.

[DUP 94] DUPUY, JP, 1994, Aux origines des sciences cognitives, Paris, La découverte.

[EDE 92] EDELMAN G.M. , 1992, Biologie de la conscience, tr.fr. Paris, Odile Jacob

[FOD 88] FODOR J \& PYLYSHYN, Z., 1988, Connectionism and cognitive architecture : a critical analysis, Cognition, $28,3-71$

[GAL 81] GALIFRET-GRANJON, N., 1981, Naissance et évolution de la représentation chez l'enfant, Paris, P.U.F.

[GAZ 87] GAZZANIGA, M., 1987, Le cerveau social, tr. fr. Paris, Robert Laffont.

[GAZ 98] GAZZANIGA, M, M., 1998, Le cerveau divisé; Pour la science, n² 251, sept 1998, p.72-79.

[HEB 49] HEBB, D.O., 1949, The Organization of Behavior, Wiley, New York.

[JOH 93] JOHNSON-LAIRD, P. N., 1993, La théorie des modèles mentaux, in Les modèles mentaux, approche cognitive des représentations, coord. M.F. Ehrlich et all., Paris, Masson

[KUH 62] KUHN, Thomas, S., 1962, La structure des révolutions scientifiques, tr.fr. 1983, Paris, Flammarion.

[LEF 85] LEFEBVRE, H., 1985, Qu'est-ce que penser ?, Paris, Publisud.

[MAT 94] MATURANA, HR. \& VARELA, FJ., 1994, L’Arbre de la connaissance, Paris, Éd. Addison-Wesley France.

[MIN 88] MINSKY, M., 1988, La société de l'esprit, Paris,InterEditions

[NEW 72] NEWEL A. \& SIMON H-A, 1972, Human Problem Solving, Prentice Hall, Englewood Cliffs, N.J.

[PIA 26] PIAGET, J., 1926, La représentation du monde chez l'enfant, réed 1993, Paris, PUF.

[PIA 45] PIAGET, J., 1945, La formation du symbole chez l'enfant, Paris, 1989, Delachaux \& Niestlé.

[PIA 55] PIAGET, J., 1955, De la logique de l'enfant à la logique de l'adolescent, Paris, PUF.

[PIA 68] PIAGET, J., 1968 a, Le structuralisme, Paris, PUF.

[PIA 70] PIAGET, J., 1970, L'épistémologie génétique, Paris, PUF. 
[PIA 58] PIAGET, J., \& MORF A., 1958, Les isomorphismes partiels entre les structures logiques et les structures perceptives, in Études d'épistémologie génétique s/d J.Piaget, tome VI Logique et Perception, Paris, PUF.

[PIA 66] PIAGET, J. \& INHELDER B., 1966, L'image mentale chez l'enfant, Paris, P.U.F. (1991).

[REU 77] REUCHLIN, M., 1977, Psychologie, Paris, PUF.

[REY 16] REY, A., 1916, Leçons de psychologie et de philosophie, Paris, Rieder et Cie.

[RIC 90] RICHARD, ,JF., 1990, Les activités mentales, Paris, Armand Colin.

[RIC 68] RICHELLE, M., 1968, Pourquoi les psychologues ?,Bruxelles, Mardaga.

[RIC 93] RICHELLE, M., 1993, Du nouveau sur l'esprit ?, Paris, PUF.

[SAL 96] SALLABERRY JC, 1996, Dynamique des représentations dans la formation, Paris, L’Harmattan (Cognition et Formation).

[SAL 02] SALLABERRY JC, 2002, Le statut de la représentation et l'articulation du niveau logique individuel et du niveau logique collectif, in Les représentations sociales - Balisage du domaine d'étude, s/d C. GARNIER ET W. DOISE, Montréal, Editions Nouvelles, p.265-278.

[SAL 96] SALLABERRY JC, 2003, Théorie de l'institution et articulation individuel-collectif, in Actualité de la théorie de l'institution, s/d ARDOINO, BOUMARD, SALLABERRY, Paris, L'Harmattan, (Cognition et Formation), p.75110.

[SAL 04] SALLABERRY JC, 2004, Dynamique des représentations et construction des concepts scientifiques, Paris, L'Harmattan, (Cognition et Formation).

[SAL 06] SALLABERRY JC, 2006, La représentation et le geste - pragmatique et représentation, Spirale, HS4 "Représentations en formation 2", p. 5-33.

[SAL 07] SALLABERRY JC, 2007, Représentation et théorie du champ, in Cognitiques $\mathrm{n}^{\circ} 11$, "La cognitique en séminaire interlabo“, p.11-22.

[SAL 15] SALLABERRY JC, 2015, La représentation: une synthèse (un concept) possible ? - Note de synthèse, L'Année de la recherche en sciences de l'éducation, $\mathrm{n}^{\circ} 2015$, p.213-256.

[SAL 17] SALLABERRY JC, 2017, «L'hypothèse de l'autonomisation des grands sous-systèmes et les risques pour la démocratie », Revue française des sciences de l'information et de la communication [En ligne], 10 | 2017, mis en ligne le 01 janvier 2017, consulté le 30 janvier 2017. URL : http://rfsic.revues.org/2823 ; DOI : 10.4000/rfsic.2823

[SEA 83] SEARLE, J.R., 1983, L'intentionnalité, tr.fr. Paris, Minuit, 1985.

[SIM 69] SIMON, H-A, 1969, The Sciences of the Artificial, tr.fr. Sciences des systèmes, sciences de l'artificiel, (tr. LE MOIGNE JL), 1991, Paris, Dunod (Afcet Systèmes)

[SIM 72] SIMON, H-A,. \& NEWELL, A., 1972, Human problem solving, Prentice Hall, Englewood Cliffs

[SMO 92] SMOLENSKY, P., 1992, IA connexionniste, IA symbolique et cerveau, in Introduction aux sciences cognitives, s/d ANDLER, Paris, Gallimard.

[VAR 89] VARELA, F.J., 1989, Autonomie et connaissance, Paris, Seuil.

[VAR 89] VARELA, F.J. 1989 b, Connaître les sciences cognitives, Paris, Seuil.

[VAR 93] VARELA, F.J. THOMPSON E. \& ROSCH E., 1993, L'inscription corporelle de l'esprit, Paris, Seuil.

[VAR 95] VARELA, F.J., 1995, Connaissances et représentations, in Dossier : Les Sciences de l'homme, B.I.C. (Bulletin d'Information des Cadres, EDF-GDF) nº 27, mars 1995, pp.78 à 88.

[VYG 85] VYGOTSKY LS., 1985, Pensée et langage, Paris, Messidor/Editions Sociales

[VYG 85] VYGOTSKY LS., 1985, Le problème de l'enseignement et du développement mental à l'âge scolaire, in Vygotsky aujourd'hui, s/d SNEUWLY \& BRONCKART, Lausanne, Delachaux \& Niestlé.

[VYG 98] VYGOTSKY LS., 1998, Traité des émotions, Paris, L'Harmattan (Cognition et Formation).

[WAL 28] WALLON, H., 1928, De l'acte à la pensée, Paris, Flammarion, 1970. 


\section{Sitographie}

[SAL 16] SALLABERRY JC, 2016, Quelle mouche a piqué Carole Barjon ?, site Educavox, onglet formation (mais on peut chercher par nom d'auteur). www. educavox.fr, EDUCAVOX - An@é 